120 $\triangle$ IEONE $\Sigma$ $\Sigma$ YNE $\Delta$ PIO TH $\Sigma$ E $\Lambda$ HNIKH $\Sigma$ ГE $\Omega \wedge O \Gamma I K H \Sigma$ ETAIPIA $\Sigma$

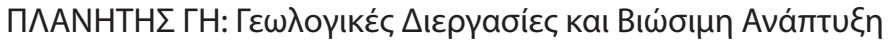

12th INTERNATIONAL CONGRESS OF THE GEOLOGICAL SOCIETY OF GREECE

PLANET EARTH: Geological Processes and Sustainable Development

\title{
ENAPKTHPIA OMIAIA
}

OPENING LECTURES 


\title{
THE “ANTHROPOCENE” IN THE MEDITERRANEAN
}

\author{
Zerefos C.S. \\ Academy of Athens \\ President, International Ozone Commission (ICSU)
}

There is ample evidence by now that climate is changing not only by natural causes but by manmade changes in the overall bio and physico-chemical balance of our planet (IPCC, 2007). This balance is maintained by interactions between the biosphere, the atmosphere, the hydrosphere and the geosphere. These four "spheres" are continuously interacting in such ways and at such rates that quasi-ensure ecological equilibrium and adaptation of humans and the ecosystems to new equilibrium states. But in the past decades, during the period called "the anthropocene", man is also contributing to climate change in a number of activities notable of which are man-made global emissions of greenhouse gases.

The man-made climate change is part of an overall global change caused by human's intervention with nature. This has significant impacts on the biological, physico-chemical systems and to the humans. The effects can be found globally distributed and they are expected to have important consequences not only to humans but to the ecosystems as well. Among the most prominent of these changes are the changes in the hydrological cycle on both global and regional scales, changes in the intensity of extreme weather events and changes in rainfall characteristics and patterns. These changes are expected to affect the incidence and severity of droughts and floods making problematic in some cases even the availability of water.

Global changes provide challenges for health and many aspects of human societies, industry and the overall wealth and security. Notable are the foreseen effects in economies, in agriculture and in food and water security. The list of negative consequences from man-made global change is indeed large. From sea level rise with severe effects on human settlements to the observed effects on biological systems. There is indeed evidence of changes and shifts in the range of plant and animal species to higher latitudes and altitudes and changes in the timing of many life-cycle events, such as flowering. This variability will affect the ecosystems and biodiversity in general. Many of these impacts, especially when operating in synergy, are expected to cause additional threats to humans, to our resources and to the ecosystems.

The year 2008 has been dedicated to Planet Earth by UNESCO and the International Union of Geological Sciences. It is a land mark year that follows the Stern review, the IPCC assessments on climate change and important international research activities in Global Change. The interactions between the hydrosphere, the atmosphere, the biosphere, the cryosphere and the geosphere have been operating on earth for millions of years in the past. Recent concerns on the effects of human activities that intervene with natural globally changing processes are based on observational, modelling and statistical analysis results. Important international efforts aim to integrate observations and coordinate international networking of modellers and experimentalists in Global Change research. 
EU and ESA have initiated the Global Monitoring of the Environment and Security (GMES) activity, recently named Kopernikus, aiming to provide to EU countries an operational service for studying man-made changes in the Environment and their related impact concerning the security of the citizens. However, the synergy between the slowly and the fast varying components of geophysical extremes, enhanced by the man-made change on the environment, is an issue of urgency, having several missing links, not considered in such an operational activity. This is particularly important when we investigate the synergistic effects of disasters resulting from nature and accelerated by man and their effects on humans, the society and the ecosystems. For example, earthquakes can be linked with landslides and tsunamis in coastal areas. The synergistic effects of these natural disasters under a manmade, globally changing environment are today mostly unknown and worst of all, there is no existing European infrastructure dealing with interactions between different types of extreme events operating in synergy under the laws of probability. The Mediterranean region is vulnerable to natural and manmade disasters. Natural disasters have always occurred in this part of the world but manmade changes to our environment have worsened the effects on humans and on the ecosystems of natural disasters.

The Mediterranean is already under pressure from global stresses and is highly vulnerable to the impacts of climate change. Floods and droughts can occur in the same area within months of each other. These events can lead to famine and widespread disruption of socio-economic wellbeing, particularly in the north African shore. Many factors contribute to the impacts of manmade climate variability in the Mediterranean and will have negative effects on its ability to cope with these changes. The overexploitation of land resources including forests, increased population, desertification and land degradation provide additional threats in this area (UNDP 2006). In parts of the Mediterranean and over the north Africa's shore, dust and sand storms have negative impacts on agriculture, infrastructure and health. The Mediterranean is also expected to face increasing water scarcity and stress. Agricultural production relies mainly on rainfall for irrigation and will be severely compromised. Under climate change some agricultural land will be lost, with shorter growing seasons and lower yields. Rising temperatures are changing the geographical distribution of disease vectors which are migrating to new areas and to higher altitudes and latitudes (WHO, 2004).

Climate change is an added stress to already threatened habitats, fragile ecosystems and species in the Mediterranean and is likely to trigger species migration and lead to habitat reduction. Land use changes due to agricultural expansion and subsequent destruction of habitat; pollution; high rates of land use change; population growth and the intrusion of exotic species. In addition, climate change is altering weather and climate patterns that previously have been relatively stable. Climate experts are particularly confident that climate change will bring increasingly frequent and severe heat waves and extreme weather events, as well as a rise in sea levels. These changes have the potential to affect human health in several direct and indirect ways, some of them severe.

Heat exposure has a range of health effects, from mild heat rashes to deadly heat stroke. Heat exposure can also aggravate several chronic diseases, including cardiovascular and respiratory disease. The results can be severe and result in both increased illness and death. Heat also increases ground-level ozone concentrations, causing direct lung injury and increasing the severity of respiratory diseases such as asthma and chronic obstructive pulmonary disease. Higher temperatures and heat waves increased demand for electricity and thus combustion of fossil fuels, generating airborne particulates and indirectly contributing to increased respiratory disease. 
Hot days and heat waves present another hazard that can act in synergy with other events. This happened for example, during the heat wave in Europe of August 2003. There were totally 40,000 deaths and this heat wave was created by meteorology; you can see the weather patterns creating extreme heat in Europe and you can see the number of deaths that were reported in Paris, Torino and Barcelona as time is proceeding and there was a very high correlation between the heat wave air temperatures and the number of deaths. The situation here was a common experience in a period of about two to three weeks and most people were elderly people that were not prepared. Usually elder people take drugs which dehydrate them. So without any signal or any information on them being dehydrated, they have been exposed to a heat wave and unintentionally of course they have been exposed to great danger for death. This can be one of the extreme or worst examples of dangers induced by synergistic effects (heat wave, high ozone levels, high dehydration, poor knowledge).

A model that has been prepared by the University of East Anglia, which shows that the heat wave of 2003 will be considered as a cooling event when compared to the year 2060 or beyond. This is because the temperature anomalies are expected to exceed about 6 degrees from now and the 2003 event was about 3 degrees above normal in the observations that have been made.

There were two more heat waves that struck our region in 2007. These heat waves (two small and one large) have set up the scene also for high risk of forest fires. Talking for the summer time, one can see that the area is also extremely vulnerable to very high levels of ozone, which is an aspiratory threat. So oxidants in the atmosphere, in addition to dehydration, in addition to the heat stress have resulted to deaths which could have been prevented; they have resulted also to setting up the scene of rare conditions for the reoccurrence of forest fires, which also operate in synergy threatening humans and the ecosystems.

Over longer time periods, increased temperatures have other effects ranging from drought to ecosystem changes that can affect health. Droughts can result in shortages of clean water and may concentrate contaminants that negatively affect the chemistry of surface waters in some areas. Drought may also strain agricultural productivity and could result in increased food prices and food shortages, worsening the situation of those affected by hunger and food insecurity. Ecosystem changes include migration of the vectors. The dynamics of disease migration are complex and temperature is just one factor affecting the distribution of these diseases.

Increased concentrations of ground-level carbon dioxide and longer growing seasons could result in higher pollen production, worsening allergic and respiratory diseases. Increased carbon dioxide concentrations in sea water may cause oceans to become more acidic and is likely to contribute to adverse ecosystem changes in the Mediterranean Sea and in the world's tropical oceans. This would have potentially dramatic implications for fisheries and the food supply in certain regions of the world.

The direct effects of extreme weather events include drowning from floods, injuries from floods, and structural collapse. Indirect effects outnumber the direct effects and likely will be more costly. Potential indirect effects include aggravation of chronic diseases due to interruptions in health care service, significant mental health concerns both from interrupted care and geographic displacement, and socioeconomic disruption resulting from population displacement and infrastructure loss.

Sea level rise increases the risk from extreme weather events in coastal areas, threatening critical 
infrastructure and worsening immediate and chronic health effects. Salt-water entering freshwater drinking supplies is also a concern for these regions, and increased salt content in soil can hinder agricultural activity in coastal areas.

Most of my examples on the effects of climate change were focused on the Mediterranean. An area of high vulnerability following the recent findings from the IPCC reports. An area where the most vulnerable populations (children and elder and particularly those suffering from other causes) are at particular risk. The Mediterranean is threatened not only by man-made and natural extreme climate and weather phenomena. It is vulnerable also to the synergy of such phenomena in case they coincide. The Mediterranean environment is by its nature fragile and vulnerable (alternating drought / floods, heat waves, seismic activity land slide threats etc.). It is fortunate that IPCC is now preparing a report on extreme phenomena and their relation to climate change. Let me express my hope that the international scientific community will also concentrate to the synergy of events and their amplified effects on humans and the ecosystems. 
